\title{
Serum lgE reduction and paradoxical eosinophilia associated with allergic conjunctivitis after dupilumab therapy
}

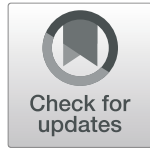

\author{
Ayaka Kimura', Ayaka Takeda', Toyo Ikebukuro ${ }^{1,2}$ and Junko Hori ${ }^{1 *}$
}

\section{To the Editor, Introduction}

Dupilumab is a fully human monoclonal antibody against the $\alpha$ subunit of the interleukin (IL)- 4 receptor (IL-4R $\alpha$ ), and inhibits IL-4 and IL-13 signalling pathways. These pathways are involved in B-cell differentiation, immunoglobulin (Ig) E production, and a Th-2dominant immune response [1]. IL-4 and IL-13 are important drivers of various atopic or allergic diseases, such as atopic dermatitis (AD) [2, 3]. Dupilumab has demonstrated efficacy and safety against multiple Th-2type inflammatory diseases including $\mathrm{AD}$ [1-3], asthma [4], chronic rhinosinusitis with nasal polyps [5] and eosinophilic oesophagitis [6] in clinical trials, and has recently been used for $\mathrm{AD}$, asthma, and chronic rhinosinusitis with nasal polyposis when existing treatments fail [7].

Adverse effects of dupilumab have been noted to include pathologies of the ocular surface, including conjunctivitis, blepharitis, keratitis, eye pruritus, dry eye, nasopharyngitis, upper respiratory tract infection, herpes simplex virus, exacerbation of $\mathrm{AD}$, injection-site infection, facial redness, alopecia, and arthralgia $[8,9]$. Biologic therapies such as dupilumab inhibit certain cytokines and suppress inflammation of the target organs, but often paradoxically induce or enhance inflammation in other organs. These phenomena are called "paradoxical reactions" [10].

Head and neck erythema have been reported as paradoxical reactions after dupilumab therapy for AD [11]. Histological examination of skin biopsies of such erythema have revealed a psoriasiform reaction pattern

\footnotetext{
* Correspondence: jhori@nms.ac.jp

'Department of Ophthalmology, Nippon Medical School Tama-Nagayama

Hospital, 1-7-1, Nagayama, Tama, Tokyo 206-8512, Japan

Full list of author information is available at the end of the article
}

suggestive of a drug-induced skin reaction [11]. The frequency of conjunctivitis in clinical trials of dupilumab and in real-world data from a systematic review and meta-analysis have been reported as $8.6-22.1 \%$ and $26.1 \%$, respectively $[8,9]$. This report presents a case of allergic conjunctivitis associated with eosinophilia as paradoxical reactions induced by dupilumab therapy for AD.

\section{Case report}

A 46-year-old woman was referred to the ocular inflammation service at Nippon Medical School TamaNagayama Hospital, for bilateral red eyes and itchiness. She was undergoing a sixth cycle of dupilumab in 10 weeks for AD. Slit lamp examination revealed bilateral conjunctive hyperemia, papillary hyperplasia, and bilateral blepharitis (Fig. 1a). No intraocular inflammation was evident in either eye. She had no past ocular history.

Blood tests revealed a high eosinophil count (1400 cells $/ \mu \mathrm{L})$ and a high concentration of $\mathrm{IgE}(8520 \mathrm{IU} / \mathrm{mL})$ at her first visit to our ocular inflammation service. Atopic dermatitis was improved after dupilumab administration, with the Investigator Global Assessment scale for Atopic Dermatitis (IGA) score improved from 4 before dupilumab administration to 2 at 10 weeks after starting dupilumab therapy. However, at the same time as improvement of dermatitis, allergic conjunctivitis paradoxically occurred. When the conjunctivitis appeared (i.e., after 10 weeks of dupilumab administration), serum IgE levels were lower than before dupilumab administration (Fig. 2a). If the allergic conjunctivitis had been a response to pollen or other antigens, serum IgE levels should have been increased, as serum IgE levels in patients with allergic conjunctivitis have been reported as high [12]. In addition, the patient had no nasal symptoms suggestive of allergic rhinitis, such as runny nose 


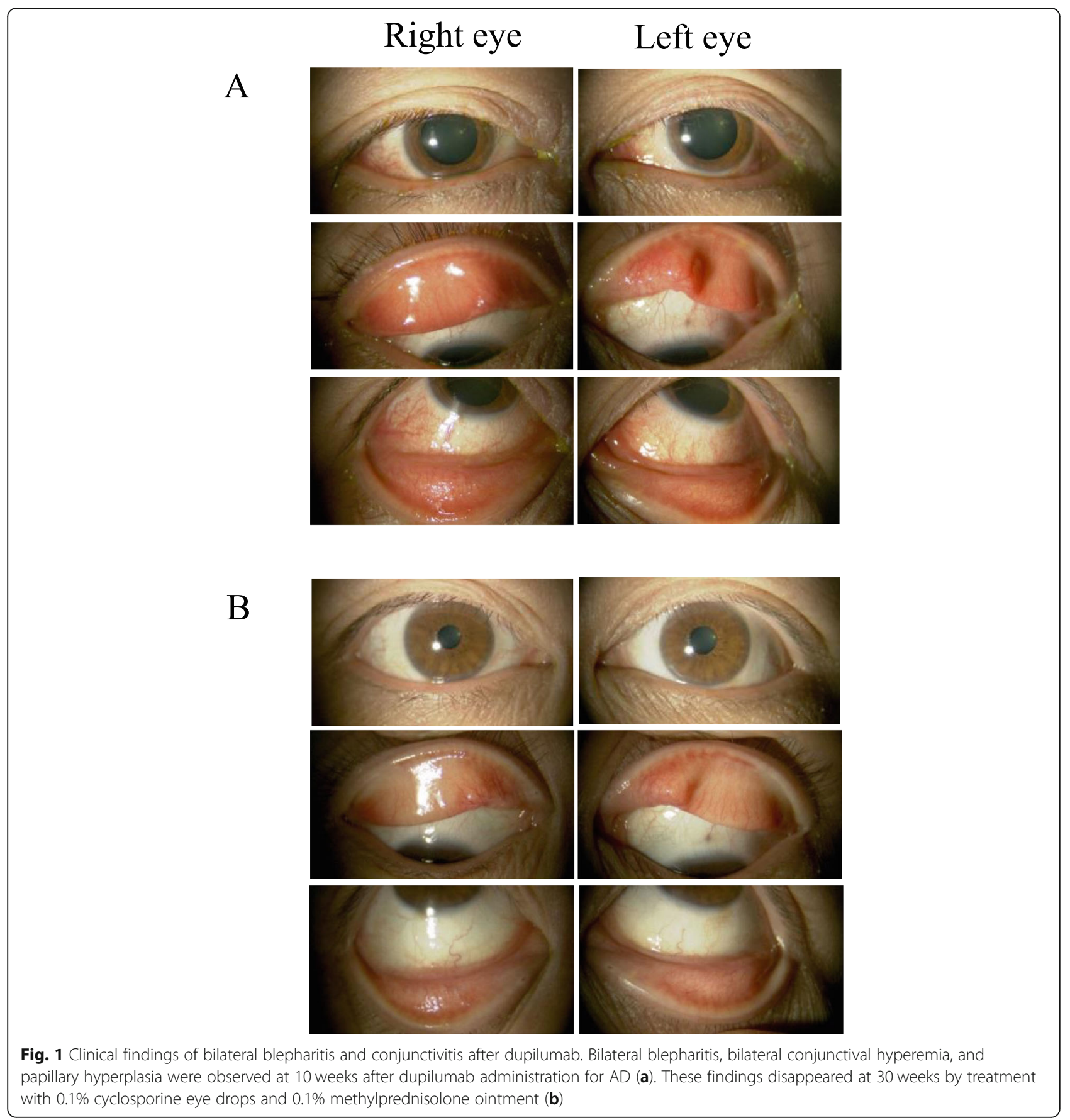

or nasal congestion. We therefore ruled out allergic conjunctivitis due to pollen or other antigens.

Allergic conjunctivitis as a paradoxical reaction induced by dupilumab was diagnosed, and she was treated with topical $0.1 \%$ cyclosporine eye drops 4 times/day and $0.1 \%$ methylprednisolone ointment twice/day for eyelids. Conjunctivitis and blepharitis gradually improved after starting these treatments, and disappeared at 30 weeks (Fig. 1b). She continued to receive dupilumab therapy, which led to decreased levels of serum
Th2-type chemokines such as thymus and activationregulated chemokine (TARC) from 470 to $241 \mathrm{pg} / \mathrm{mL}$ by 34 weeks after dupilumab therapy, indicating reduced severity of AD.

It is worth noting that laboratory data showed inverse changes in eosinophils and IgE before and after starting dupilumab (Fig. 2). IgE levels decreased markedly from $23,100 \mathrm{IU} / \mathrm{mL}$ at 3 weeks before to $8520 \mathrm{IU} / \mathrm{mL}$ at 10 weeks after dupilumab administration (Fig. 2a). On the other hand, eosinophil count paradoxically increased 

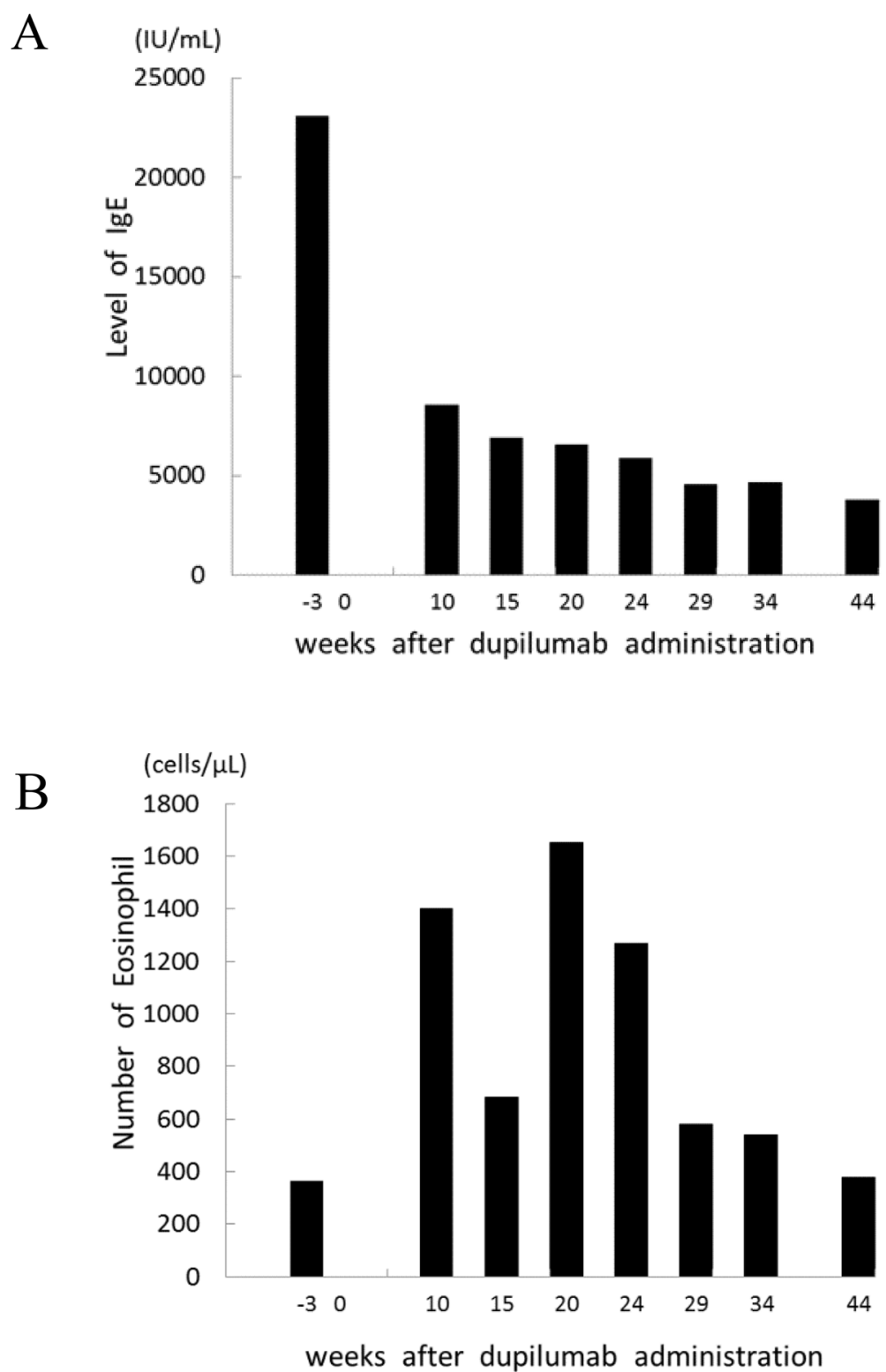

Fig. 2 Decreased levels of IgE and paradoxical transient eosinophilia in peripheral blood after dupilumab therapy. IgE level decreased markedly from $23,100 \mathrm{IU} / \mathrm{mL}$ at 3 weeks before to $8520 \mathrm{IU} / \mathrm{mL}$ at 10 weeks after dupilumab administration (a). The number of eosinophils paradoxically increased from 365 cells/ $\mu \mathrm{L}$ at 3 weeks before to 1400 cells/ $\mu \mathrm{L}$ at 10 weeks after dupilumab administration (b)

from 365 cells $/ \mu \mathrm{L}$ to 1400 cells $/ \mu \mathrm{L}$ (Fig. $2 \mathrm{~b}$ ). Conjunctivitis occurred concomitant with eosinophilia.

\section{Discussion}

Dupilumab has been reported to induce eosinophilia with a peak at around 4 weeks after administration [13]. In clinical trials of dupilumab for AD patients, 155 of 465 patients showed eosinophilia at 4 weeks and 3 of 465 patients showed Grade 3 eosinophilia $\left(>5 \times 10^{9}\right.$ cells/L) [13]. Inhibition of eosinophil recruitment from peripheral blood to inflamed skin tissues by dupilumab has been reported as a mechanism underlying increased eosinophils in peripheral blood [13].

Eosinophils are major pathogenic immune cells in allergic conjunctivitis [14]. Dupilumab-mediated eosinophilia as a paradoxical reaction may be one of the potential mechanisms behind allergic conjunctivitis induced by dupilumab [14]. Further studies are needed to evaluate whether dupilumab-mediated eosinophilia led to eosinophil infiltration into the conjunctiva. Risk factors for the development of dupilumab-associated conjunctivitis have been reported to include AD severity, history of conjunctivitis and elevated levels of 
biomarkers such as TARC, IgE, and eosinophil counts in peripheral bood [8]. The patient in this report was obviously a high-risk patient, showing high AD severity with an Investigator Global Assessment scale for Atopic Dermatitis score of 4 and elevated levels of both IgE and eosinophils.

\section{Conclusion}

We examined serum IgE, eosinophil counts, and clinical findings of conjunctivitis after dupilumab therapy for more than 6 months in a single patient. This is the first report to describe serum IgE reduction and eosinophilia occurred simultaneously in a patient with allergic conjunctivitis associated with paradoxical eosinophilia after dupilumab therapy. Topical cyclosporine eye drops and steroid ointment offered significant efficacy against dupilumab-associated conjunctivitis, and enabled the $\mathrm{AD}$ patient to continue dupilumab therapy.

\section{Abbreviations}

IL: Interleukin; IL-4Ra: Interleukin-4 receptor alpha; Ig: Immunoglobulin; AD: Atopic dermatitis; TARC: Thymus and activation-regulated chemokine

\section{Acknowledgements}

The authors would especially like to acknowledge Dr. Naoyuki Azuma, at Department of Dermatology, Nippon Medical School Tama-Nagayama Hospital for his support.

\section{Authors' contributions}

AK contributed to the data collection, review of literature, and writing the manuscript. AT and $\mathrm{TI}$ curated the patient data. JH interpreted the patient data, and contributed in supervision, writing, and editing the manuscript. All authors read and approved the final manuscript.

\section{Funding}

This work was supported from the Grant-in-Aid for Scientific Research from Japan Society for the Promotion of Science 20 K09813(J.H.). The role of the funding is writing and English proofreading.

\section{Availability of data and materials}

Not applicable.

\section{Ethics approval}

The 'Nippon Medical School Tama Nagayama Hospital' 'Ethics Committee of Tama Nagayama Hospital' confirmed that ethics approval was not required.

\section{Consent for publication}

Written informed consent was obtained from the patient for publication of her details and accompanying images in this manuscript.

\section{Competing interests}

The authors declare that they have no competing interests.

\begin{abstract}
Author details
'Department of Ophthalmology, Nippon Medical School Tama-Nagayama Hospital, 1-7-1, Nagayama, Tama, Tokyo 206-8512, Japan. ²Department of Ophthalmology, Nippon Medical School Hospital, 1-1-5, Sendagi, Bunkyo, Tokyo 113-8603, Japan.
\end{abstract}

Received: 6 September 2020 Accepted: 27 December 2020 Published online: 15 February 2021

\section{References}

1. Simpson EL, Bieber T, Guttman-Yassky E et al (2016) Two phase 3 Trials of Dupilumab versus Placebo in Atopic Dermatitis. N Engl J Med 375:23352348
2. Beck LA, Thaçi D, Hamilton JD et al (2014) Dupilumab treatment in adults with moderate-to-severe atopic dermatitis. N Engl J Med 371:130-139

3. Thaçi $D$, Simpson EL, Beck LA et al (2016) Efficacy and safety of dupilumab in adults with moderate-to-severe atopic dermatitis inadequately controlled by topical treatments: a randomised, placebo-controlled, dose-ranging phase $2 \mathrm{~b}$ trial. Lancet 387:40-52

4. Castro M, Corren J, Pavord I et al (2018) Dupilumab efficacy and safety in moderate to severe uncontrolled asthma. N Engl J Med 378:2486-2496

5. Bachert C, Mannent L, Naclerio RM et al (2016) Effect of subcutaneous dupilumab on nasal polyp burden in patients with chronic sinusitis and nasal polyposis: a randomized clinical trial. JAMA 315:469-479

6. Hirano I, Dellon ES, Hamilton JD et al (2017) Dupilumab efficacy and safety in adult patients with active eosinophilic oesophagitis: a randomised double-blind placebo controlled phase 2 trial. United Eur Gastroenterol J 5: $1138-1150$

7. Sanofi and Regeneron Pharmaceuticals, Inc. (2020) Take Action With DUPIXENT (dupilumab). https://www.dupixent.com/ Accessed 8 Aug 2020

8. Akinlade B, Guttman-Yassky E, Bruin-Weller DM et al (2019) Conjunctivitis in dupilumab clinical trials. Br J Dermatol 181:459-473

9. Halling AS, Dyrberg LN, Silverberg Jl et al (2020) Real-world evidence of dupilumab efficacy and risk of adverse events: a systematic review and meta-analysis. J Am Acad Dermatol S0190-9622(20):32442-32447

10. Ramos-Casals M, Roberto PA, Diaz-Lagares C et al (2010) Autoimmune diseases induced by biological agents. A double-edged sword? Autoimmun Rev 9:188-193

11. Wijs de LEM, Nguyen NT, Kunkeler ACM, Nijsten T et al (2019) Clinical and histopathological characterization of paradoxical head and neck erythema in patients with atopic dermatitis treated with dupilumab: a case series. In: $\mathrm{Br} J$ Dermatol

12. Mimura T, Usui T, Mori M et al (2011) Relationship between total tear and serum IgE in allergic conjunctivitis. Int Arch Allergy Immunol 154(4):349-352

13. Wollenberg A, Beck LA, Blauvelt A et al (2020) Laboratory safety of dupilumab in moderate-to-severe atopic dermatitis: results from three phase III trials. (LIBERTY AD SOLO1, LIBERTY AD SOLO2, LIBERTY AD CHRONOS). Br J Dermatol 182:1120-1135

14. Thyssen JP, Toft PB, Halling-Overgaard AS, Gislason GH, Skov L, Egeberg A (2017) Incidence, prevalence, and risk of selected ocular disease in adults with atopic dermatitis. J Am Acad Dermatol 77:280-286

\section{Publisher's Note}

Springer Nature remains neutral with regard to jurisdictional claims in published maps and institutional affiliations.

\section{Submit your manuscript to a SpringerOpen ${ }^{\circ}$ journal and benefit from:}

- Convenient online submission

- Rigorous peer review

- Open access: articles freely available online

High visibility within the field

- Retaining the copyright to your article

Submit your next manuscript at $\boldsymbol{\nabla}$ springeropen.com 